(C) 2010 IEEE. Reprinted, with permission, from Fabra, F., Cardellach, E., Nogués, 0., Oliveras, S., Ribó, S., Rius, A., Belmonte, M., Semmling, M., Macelloni, G., Pettinato, S., Zasso, R., D Addio, S., Monitoring sea ice and dry snow with GNSS reflections, Proceedings of IEEE International Geoscience and Remote Sensing Symposium (IEEE IGARSS), and july/2010.

This material is posted here with permission of the IEEE. Such permission of the IEEE does not in any way imply IEEE endorsement of any of CSIC products or services. Internal or personal use of this material is permitted. However, permission to reprint/republish this material for advertising or promotional purposes or for creating new collective works for resale or redistribution must be obtained from the IEEE by writing to pubs-permissions@ieee.org.

By choosing to view this document, you agree to all provisions of the copyright laws protecting it. 


\title{
MONITORING SEA-ICE AND DRY SNOW WITH GNSS REFLECTIONS
}

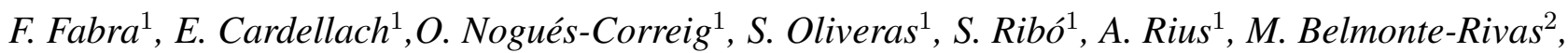

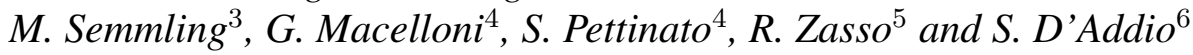 \\ ${ }^{1}$ Institut de Ciències de l'Espai (ICE-IEEC/CSIC), Spain, ${ }^{2}$ KNMI, Netherlands \\ ${ }^{3}$ GFZ, Germany, ${ }^{4}$ IFAC-CNR, Italy, ${ }^{5}$ CVA-ARPAV, Italy, ${ }^{6}$ ESA-ESTEC, Netherlands
}

\begin{abstract}
GPS reflected signals have become a source of opportunity for remote sensing of the Earth's suface. In this work, we present several capabilities of this technique in two different polar environments: Greenland and Antarctica. The first part is dedicated to the retrieval of sea-ice properties, giving emphasis to the study of the coherent phase for altimetric and roughness estimations, and polarimetric measurements for the determination of the ice salinity variation. The results show good agreement with a tide model and daily ice charts. On the second part, some preliminary results and analysis strategies to retrieve dry snow signatures are presented.
\end{abstract}

Index Terms - GNSS-R, sea-ice, dry snow, bistatic radar, altimetry

\section{INTRODUCTION}

This work began in the frame of the GPS-SIDS project (funded by ESA and conducted by ICE-IEEC/CSIC, GFZ, IFAC/CNR, ADT), whose objective was to investigate the viability of using reflected GPS signals to study sea-ice and dry snow properties from space. For that purpose, it was required to obtain relatively long term high quality data sets using ground-based platforms and then extrapolate the obtained results to a spaceborne platform. The logistic constraints in the Polar environments make this task specially challenging. The project was divided into two parts depending on the type of measurement (sea-ice and dry snow) and two different scenarios were selected.

The concept of GNSS-R (GNSS-Reflectometry) or PARIS (Passive Reflectometry and Interferometry System) was suggested in 1993 [1] as a remote sensing technique based on the analysis of reflected L-band GNSS signals (bistatic radar). This approach has been motivated by the availability of the GPS, GLONASS and future GALILEO constellations of navigation satellites. Despite of being initially conceived as a means toward sea surface altimetry, GNSS reflections offer

This work has been funded by ESA C.N. 21793/08/NL/ST and AYA2008-05906-C02-02/ESP Spanish grant many other potential applications, such as ocean wind speed [2], soil moisture changes [3], sea surface state determination [4], and sea ice detection and classification [5].

The system responsible of acquiring direct and reflected GPS signals has been the GPS Open Loop Differential Real Time Receiver (GOLD-RTR [6]). It was designed, developed and tested at IEEC/CSIC with the aim of collecting GNSS signals reflected off the Earth's surface from up to three radio front-ends, and generate the complex cross-correlation function (waveform) in real-time. The instrument has been widely used since 2005 [7, 8], and nowadays it has been replicated (3 additional GOLD-RTRs available).

\section{THE EXPERIMENT SITES}

The first part of the project (GPS - Sea Ice) started at the end of October 2008, taking place at Godhavn (Qeqertarsuaq), Latitude $69^{\circ} \mathrm{N}$, Greenland. The equipment was installed at a telecommunication tower situated at the edge of a cliff at approximately 650 meters above sea level. The orientation of the site points the azimuthal field of view over the sea towards the South, where most of the GPS constellation lies at these latitudes. Due to the coastline profile, the range of elevations for GPS signals reflected off the sea surface goes from $1^{\circ}$ to $15^{\circ}$. Under this conditions, our instrument was able to monitor continuously the complete process of formation, evolution and melting of sea-ice until mid May 2009, summing up to 1.4 TBs of data.

The second part of the project (GPS - Dry Snow) was carried on in December 2009. The Italian-French base of Concordia, located at Dome $\mathrm{C}$ in the middle of the East Antarctic plateau (Latitude $75^{\circ} \mathrm{S}$ ), was the selected scenario for this experiment. The antennas of the GOLD-RTR system were installed on the top of a $45 \mathrm{~m}$ high tower pointed toward to a dry snow protected area. Starting on December $10^{t h}$, more than one week of raw-data waveforms were collected. The experience of several past campaigns (e.g. bedrock and snow topography, snow accumulation rate, etc.) as well as the different instruments developed in-situ for continuous atmospheric and snow measurements make this area very attractive for the cal- 
ibration of any remote sensing mission. Regarding L-band signals, a pilot ground experiment called DOMEX was conducted during the Austral summer of 2004-2005 [9]. This experiment, that included radiometric L- and C- band measurements from a tower at different incidence and azimuth angles and snow measurements, confirmed the spatial uniformity (on a one-kilometer scale) and temporal stability (on a monthly scale) of the snow layers emitting low frequency microwave radiation. In order to verify the emission stability over a longer period, a new experiment (called DOMEX-2) started in December 2008 and it will be concluded in December 2010 .

\section{REMOTE SENSING OF THE SEA-ICE}

The sea-ice is typically characterized by its concentration, thickness, roughness, and presence of salinity. Different classifications exist, such as the percentage coverage (part of the surface covered by sea-ice); the form of the ice (size of the floes: pancake, growler, floe...); and the stage of development (thickness and age of the ice). The GNSS technique can potentially measure the sea-ice altimetry, which relates to the free-board parameter (altitude between the floating line and the free top surface), which in turn is linked to the total thickness. GNSS-R can also infer roughness properties, which should help characterizing the ice type, together with the presence of salinity. GNSS-R estimates of sea-ice types have been tackled in [5], by means of the total reflected power (linked to permittivity) and waveform shape (roughness). Those works were conducted from aircrafts, and unlike our campaign, they used small incidence angles of observation. In the following subsections we discuss some other techniques applied to sea-ice reflected data for altimetric and salinity retrieval capabilities.

\subsection{Study of the phase}

Observations done by UKDMC satellite [10] indicated the potential use of the phase coherence of the reflected GPS signals from sea-ice. As time goes by and the GNSS satellite moves across the sky, the incidence angle of the observation changes, and with it, the delay between the direct and reflected signals. This delay $\rho$ depends on the altitude of the receiver above the reflecting surface $H$, as $\rho=2 H \sin (e)$ being $e$ the elevation angle (complementary to the incidence, $90^{\circ}-\theta_{\text {inc }}$ ). Therefore, the evolution of the differential phase between direct and reflected signal could estimate the height of the receiver with respect to the specular surface. However, signal fading and multipath make this process difficult. To solve it, the approach followed has been to stop the phase by counterrotating the complex IQ vector with a combination of both geometric and atmospheric modeled delays. The IQ vector is obtained by multiplying the complex components from the peak of the reflected waveform with the conjugated equivalents from the

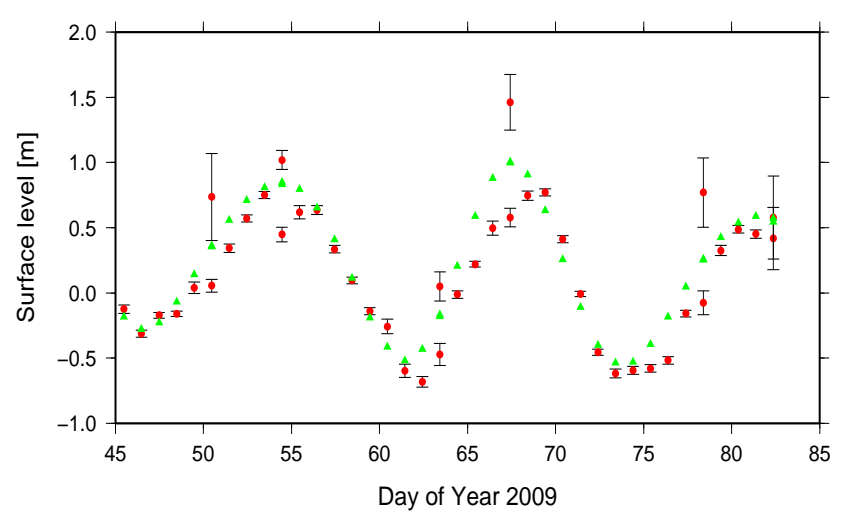

Fig. 1. Comparison between surface level estimation given by the differential phase from PRN 20 (red dots) with the Artic Tide Model AODTM-5 (green triangles).

direct signal. The geometric delay is computed as the relative distance between the path that goes from the satellite to the specular point and continues to the receiver, with respect to the satellite-to-receiver direct path. The position of both ends is known and the specular is computed over the geoid EGM96. The atmospheric delay is given by a global mapping function [11] and the Total Zenith Delay provided by GFZ. Once the differential phase is stopped, a linear fit is applied to estimate a residual height $(\Delta H)$, which can be interpreted as an estimation of the surface level. Fig. 1 shows an example of this estimation and compares it with the Artic Tide Model AODTM-5 [12] computed at the same time of the day. The comparison shows good agreement. Moreover, as the roughness of the surface increases, the signal loses coherence and becomes noisier. Fig. 2 shows the root mean square of the stopped phase (in $\mathrm{cm}$ ) as a function of $\sin (e)$ (image on the left) for a determined satellite and several days. The image on the right side of the figure shows the ice concentration provided by daily ice charts from DMI, and interpolated to the locations of the specular points. A qualitative matching between the shape of both plots is noticeable.

\subsection{Polarimetric analysis}

The presence of salinity in the sea ice modifies its dielectric properties, resulting in different amplitude and phase for the co- and cross-polar components of the complex Fresnel coefficients. This phase difference is captured as the POlarimetric Phase Interferometry (POPI) [13], the phase of the complex conjugate of the received co- and cross-polarized fields $E_{r}^{R H C P} E_{r}^{L H C P *}$. The GOLD-RTR is not able to provide the absolute phases, because of the arbitrary phase used to set up each correlation channel at the beginning of a track-record. Nevertheless, variations in the POPI might relate to variations in the ice salinity. Similarly, the Polarization Ratio between the amplitude of both components (RHCP/LHCP) should also 

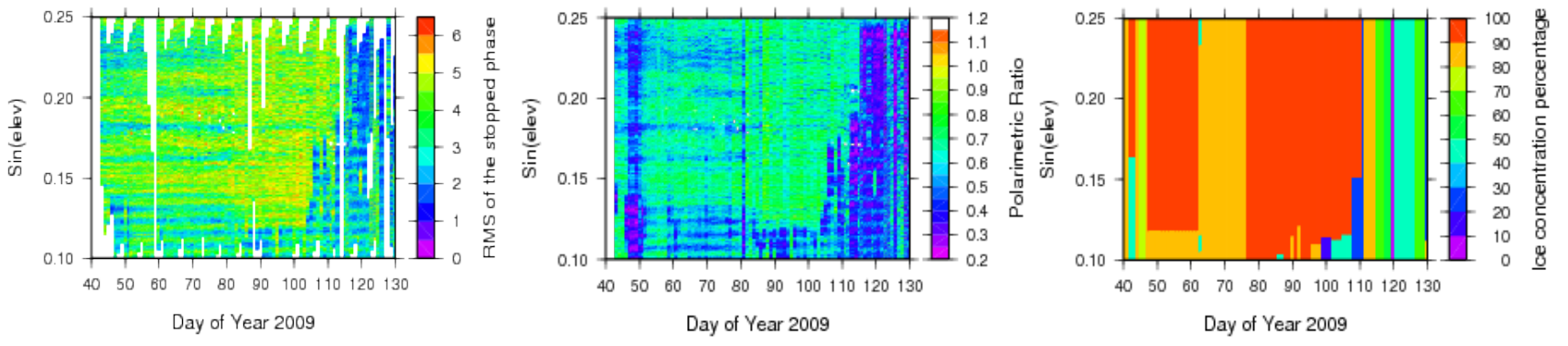

Fig. 2. Different plots as a function of $\sin (e)$ for several days: (Left) RMS of the stopped differential phase from PRN 02; (Center) Polarization Ratio (RHCP/LHCP) from PRN 02; (Right) Ice concentration provided by DMI.

relate to ice salinity variations. Fig. 2 shows this ratio computed with real data, as a function of $\sin (e)$ (center image) for a determined satellite and several days. Again, there is also a qualitative matching with the shape of the ice concentration.

\section{REMOTE SENSING OF THE DRY SNOW}

The relative transparency of continental ice to radar waves with frequencies under $1000 \mathrm{MHz}$ has been widely used to investigate the internal properties of continental ice masses [14], where maximum depths of about $4000 \mathrm{~m}$ in cold ice and about $1500 \mathrm{~m}$ in temperate ice have been sounded. The vertical structure or internal dielectric layering within the ice sheet is usually inferred from the time delay suffered by distinct echoes reflected off boundaries separating media with different dielectric properties.

GPS signals, with frequencies above the $1000 \mathrm{MHz}(1200-$ $1500 \mathrm{MHz}$ ), will have smaller penetration depth than lower frequencies -typically 100 meters penetration-, which relates to millennium scale accumulation rate [15]. Nevertheless, the proximity of these frequencies to the SMOS and Aquarius L-band radiometers $(1400 \mathrm{MHz})$, will contribute to the understanding of radiometric measurements, by revealing information about the internal layer information of dry snow at Antarctica. The Ground-Penetrating Radars (GPS, $100 \mathrm{KHz}$ to $1 \mathrm{GHz}$ frequencies) show layers at 10-20 meter spacing.

\subsection{Code delay analysis}

Numerical simulations of snow sub-structure and volumetric scattering have been shown in [15], using the GPS P-code signals. The method presented in that paper uses volumetric scattering based in multi-layer reflections modeled in geometrical optics limit of the Kirchhoff approximation. Unfortunately, only the C/A code has been available during the Dry-Snow campaign, of ten times coarser delay resolution.

Figure 3 shows an example of a time series of reflected waveforms (1 sec of uncoherent integration) gathered at Dome-C. In this scenario, The GOLD-RTR was configured to have the peak of the reference (direct) signal at lag 22 . It

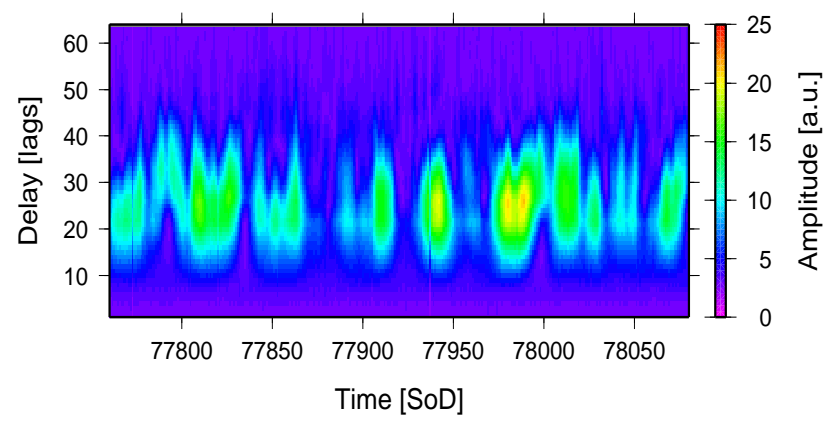

Fig. 3. Time series of reflected LHCP waveforms (1 sec of uncoherent integration) from PRN 11 on Dec $19^{\text {th }}$ at DomeC.

can be seen how the peak of the acquired waveform, which is related to the specular reflection, varies its amplitude and position with respect to lag 22 . No significant patterns have been found with respect to elevation/azimuth that may be related to a close multipath. With the delay $\rho$ obtained from this position, we have computed the vertical distance $H$ from the receiver by using $\rho=2 H \sin (e)$. The histograms of these estimations are shown in figure 4 for two different days. Despite that most of the values lie at the surface level (45 meter under the antenna's position), the results show several estimations referring to internal layers. Values close to zero are due to the effect of the direct signal and the failure of the delay model employed at low elevations, whereas values below $45 \mathrm{~m}$ might also be contaminated by leakages of direct signal.

This simple analysis has only taken into account the average contribution from all possible reflections. For this reason, we plan to complement the previously mentioned multi-layer method with polarimetric and phase information of the waveform. The GOLD-RTR delay-lag resolution (15 meter interlag resolution) might be able to resolve the snow layer substructure if phase and polarimetric signatures are found. To attempt to tackle the contributions of these internal reflections iteratively, by modeling the complex waveform as the sum of a finite number of multi-path reflections separated both delay 
and phase will be a topic for future work.

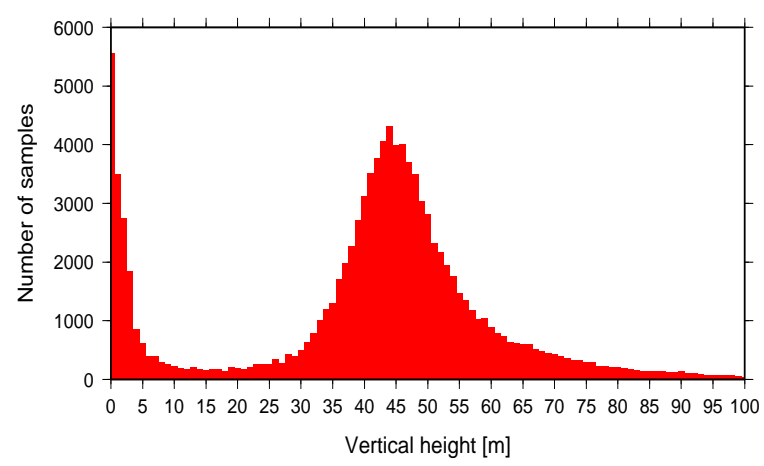

December $18^{t h}$

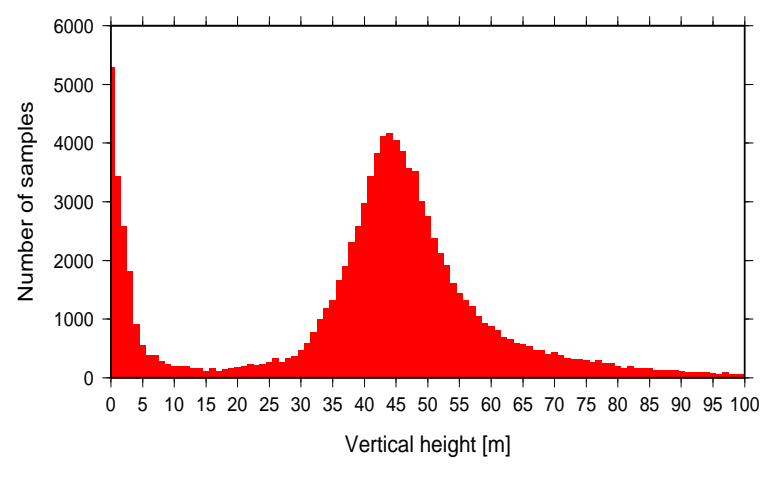

December $20^{t h}$

Fig. 4. Histograms of the estimated vertical distance between the receiving antenna and the reflections' layer. Only LHCP reflections have been employed and signals with low SNR have been filtered out.

\section{REFERENCES}

[1] M. Martín-Neira, "A passive reflectometry and interferometry system (PARIS): application to ocean altimetry," ESA Journal, vol. 17, pp. 331-355, 1993.

[2] J.L. Garrison, A. Komjahty, V.U. Zavorotny, and S.J. Katzberg, "Wind speed measurements using forward scattered GPS signals," IEEE Trans. Geosci. and Remote Sens., vol. 40, no. 1, pp. 50-66, 2002.

[3] D. Masters, V.U. Zavorotny, S.J. Katzberg, and W. Emery, "GPS signal scattering from land for moisture content determination," in Proc. IEEE IGARSS 2000, Honolulu, HI, USA, July 2000.

[4] A. Rius, J.M. Aparicio, E. Cardellach, M. Martín-Neira, and B. Chapron, "Sea surface state measured using GPS reflected signals," Geophysical Research Letters, vol. 29, no. 23, December 2002.
[5] M. Belmonte, J. A. Maslanik, and P. Axelrad, "Bistatic scattering of GPS signals off Arctic sea ice," IEEE Trans. Geosci. and Remote Sens., vol. 48, 2009.

[6] O. Nogués-Correig, E. Cardellach Galí, J. Sanz Campderròs, and A. Rius, "A GPS-Reflections Receiver That Computes Doppler/Delay Maps in Real Time," IEEE Trans. Geosci. and Remote Sens., vol. 45, no. 1, pp. 156-174, January 2007.

[7] E. Cardellach and A. Rius, "A new technique to sense non-Gaussian features of the sea surface from L-band bi-static GNSS reflections," Remote Sensing of Environment, vol. 112, pp. 2927-2937, 2008.

[8] A. Rius, E. Cardellach, and M. Martín-Neira, "Altimetric Analysis of the Sea Surface GPS Reflected Signals," IEEE Trans. Geosci. and Remote Sens., 2009.

[9] G. Macelloni et al., "DOMEX 2004: An Experimental Campaign at Dome-C Antarctica for the Calibration of Spaceborne Low-Frequency Microwave Radiometers," IEEE Trans. Geosci. and Remote Sens., vol. 44, 2006.

[10] S. Gleason et al., "Detection and processing of bistatically reflected GPS signals from low earth orbit for the purpose of ocean remote sensing," IEEE Trans. Geosci. and Remote Sens., vol. 43, 2005.

[11] J. Boehm, A.E. Niell, P. Tregoning, and H. Schuh, "Global Mapping Functions (GMF): A new empirical mapping function based on numerical weather model data," Geophysical Research Letters, vol. 33, 2006.

[12] L. Padman and S. Erofeeva, "A barotropic inverse tidal model for the Arctic Ocean," Geophysical Research Letters, vol. 31, 2004.

[13] E. Cardellach, S. Ribó, and A. Rius, "Technical Note on POlarimetric Phase Interferometry (POPI)," Arxiv preprint physics/0606099, 2006.

[14] L.A. Plewes and B. Hubbard, "A review of the use of radioecho sounding in glaciology," Progress in Physical Geography, vol. 25, 2001.

[15] M. Wiehl, R. Légrésy, and R. Dietrich, "Potential of reflected GNSS signals for ice sheet remote sensing," Progress in Electromagnetics Research, vol. 40, pp. 177-205, 2003. 